\title{
Fracture Behavior Investigation of the Propellant Material Using the Extended Finite Element Method
}

\author{
Li ZHANGa ${ }^{a}$, Jin-Zhao HUANG ${ }^{\text {b* }}$ and Zhi LVc \\ Department of Astronautic Science and Mechanics, Harbin Institute of Technology, China \\ a19666894@hit.edu.cn, bhjz987622184@sina.com \\ ${ }^{*}$ Corresponding author: Jin-Zhao Huang
}

Keywords: Crack propagation process, Extended FINITE ELEMENT METHOD (XFEM), Propellant material

Abstract. When simulating crack propagation process using the finite element method, we need to create singularity grids at the crack tip. And with the increase in crack length, we need a re-division of grids. This makes the calculation very complicated. The extended finite element method (XFEM) is an effective method for simulating crack extension. And the crack is independent of the grids. Because there is no complicated grid at the crack tip in the XFEM model, the computation efficiency can be increased greatly. This paper investigated the fracture behavior of the propellant material with an initial crack using XFEM. By comparing with existing experimental results, this paper shows that XFEM can be used to simulate fracture characteristic of the propellant material efficiently.

\section{A Brief Introduction of XFEM}

As shown in Figure.1, there exists an arbitrary crack in the grids.

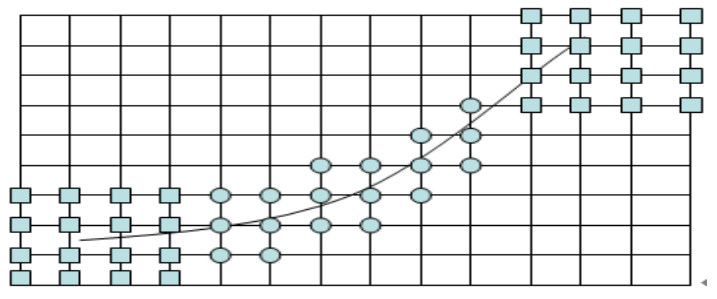

Figure.1 Grids with an arbitrary crack

In order to control the influence of the crack on the displacement, we strengthen the node degree of freedom around the crack. Then through interpolation function, the displacements of any point around the crack can be obtained.

$$
\left\{\begin{array}{l}
u^{n}(x) \\
v^{n}(x)
\end{array}\right\}=\sum_{i \in \Omega} N_{i}(x)\left\{\begin{array}{l}
u_{i} \\
v_{i}
\end{array}\right\}+\sum_{i \in \Omega_{r}} N_{i}(x) H(x)\left\{\begin{array}{l}
a_{1 j} \\
a_{2 j}
\end{array}\right\}+\sum_{i \in \Omega_{\Lambda}} N_{i}(x)[L]\left\{\begin{array}{c}
u_{m}^{n p} \\
v_{m}^{n p}
\end{array}\right\}
$$

Among them, $H(x)$ is a jump function:

$$
H\left(x^{\prime}, y^{\prime}\right)=\left\{\begin{array}{c}
1\left(y^{\prime}>0\right) \\
-1\left(y^{\prime}<0\right)
\end{array}\right.
$$

$N_{i}$ is nodal shape function. ${ }^{\Omega_{r}}$ is the mesh region with crack. ${ }^{\Omega_{\Lambda}}$ is the mesh region of the crack tip. $\Omega$ is the whole mesh region. $\left\{\begin{array}{l}u_{i} \\ v_{i}\end{array}\right\}$ is node displacement. $\left\{\begin{array}{l}a_{1 j} \\ a_{2 j}\end{array}\right\}$ is strengthened degree of freedom related to the jump function. $\left\{\begin{array}{l}u_{m}^{n p} \\ v_{m}^{n p}\end{array}\right\}$ is the incremental displacement of the crack tip. $[L]$ is transformation matrix between global coordinate system and local coordinate of the crack tip. 
Under local coordinate systems, the formula of mixed mode incremental displacement in the crack tip is shown as follows:

$$
\left\{\begin{array}{l}
u^{i n p} \\
v^{i n p}
\end{array}\right\}=\sum_{n=1}^{M}\left(\begin{array}{ll}
f_{11 n} & f_{12 n} \\
f_{21 n} & f_{22 n}
\end{array}\right)\left\{\begin{array}{l}
K_{1 n} \\
K_{2 n}
\end{array}\right\}
$$

Here, $K_{1 n}$ and $K_{2 n}$ are coefficients. $K_{11}$ is the mode-I stress intensity factor. $K_{21}$ is the mode-II stress intensity factor.

After constructing the displacement model, the basic control equations can be deduced by the principle of virtual work using conventional finite element method.

\section{The Fracture Criterion Of The Propellant Material}

Fracture criterion should be inputted to judge the crack propagation direction when using XFEM to calculate crack propagation process. Because very few fracture criterions can be found for the propellant material, this paper will find out the fracture criterion of this special material. We will use a tensile test model (Figure.2) with the length $115 \mathrm{~mm}$, width $48 \mathrm{~mm}$, thickness $5 \mathrm{~mm}$, crack length $10 \mathrm{~mm}, \beta=45^{\circ}$ given in Ref. [4]. It`s force-displacement curve is shown as Figure.3.

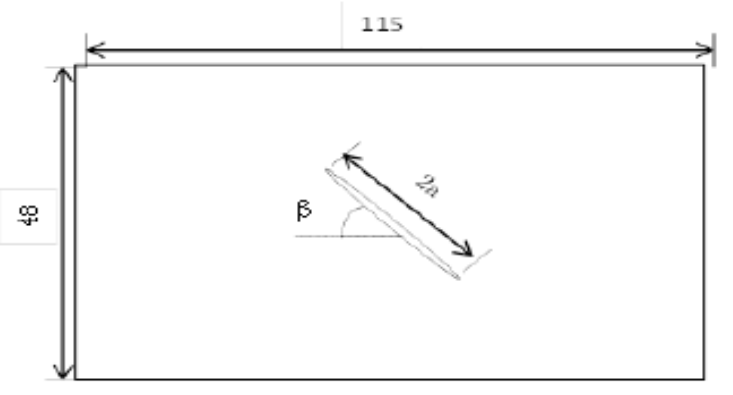

Figure.2 The size of tensile test

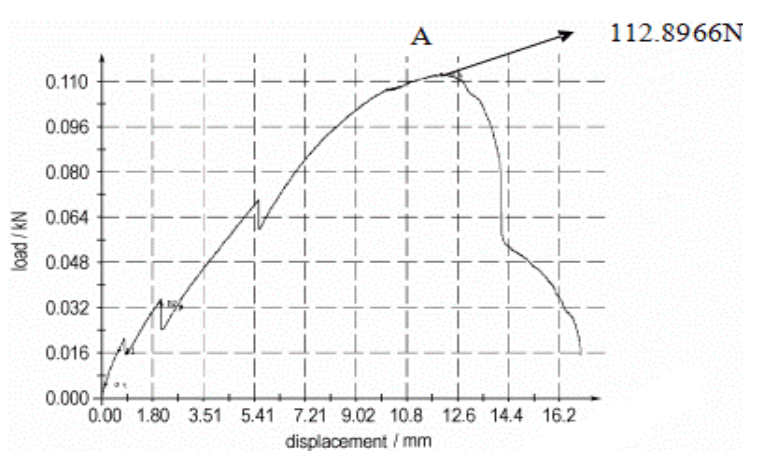

Figure.3 The force-displacement curve [4]

It can be seen that the tension increased gradually with the strain before A point. After A point the tension dropped suddenly. It shows that the crack was unstable after A point. The tension was measured as $112.8966 \mathrm{~N}$. In the simulation process, we will apply the tension on the plate stenosis surface averagely. This average tension will be used in this finite element calculation.

\section{Finite Element Model}

The model size, the crack`s length, location and direction are same to the real test when building the finite element method. The number of grid units is 1820 . The calculation model, loading condition and grids are given as shown in Figures.4-6.

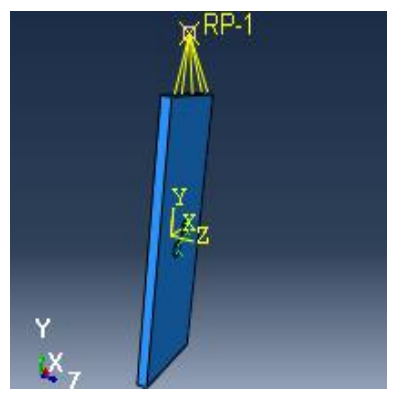

Figure.4 The Coupling of tension

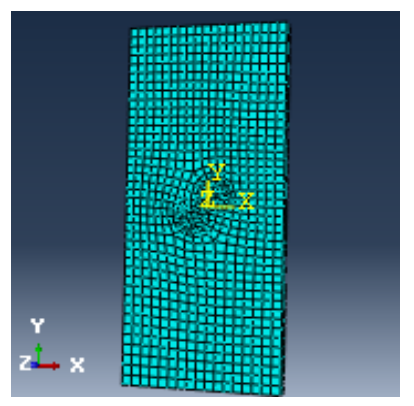

Figure.5 Global meshing of grids 


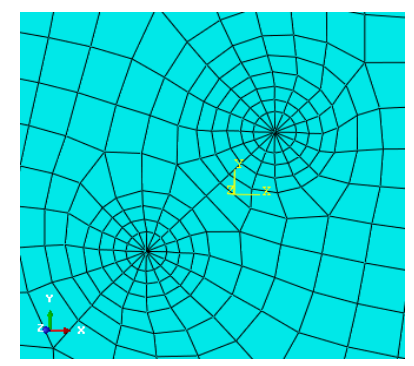

Figure.6 Meshing near the crack tip

\section{Calculation Results}

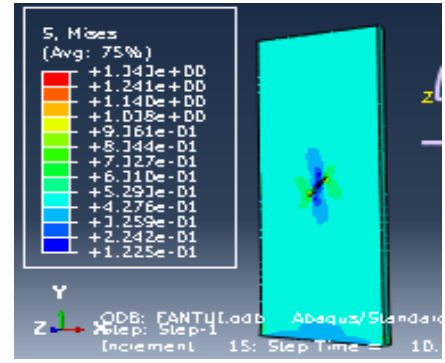

Figure.7 Global stress distribution

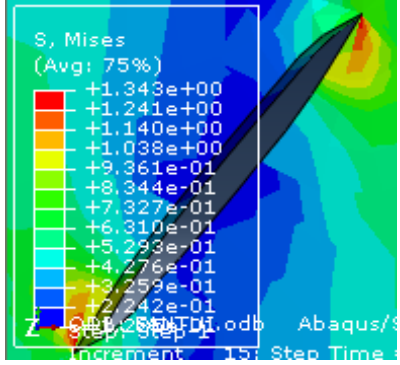

Figure.8 Crack tip stress distribution

Through post-processing we can get the maximum principal stress is $1.913 \mathrm{MPa}$. It will be used as the input item of the fracture criterion when using XFEM in this paper. The displacement around crack tip is $0.2521 \mathrm{~mm}$. It is the displacement input item in the evolution item.

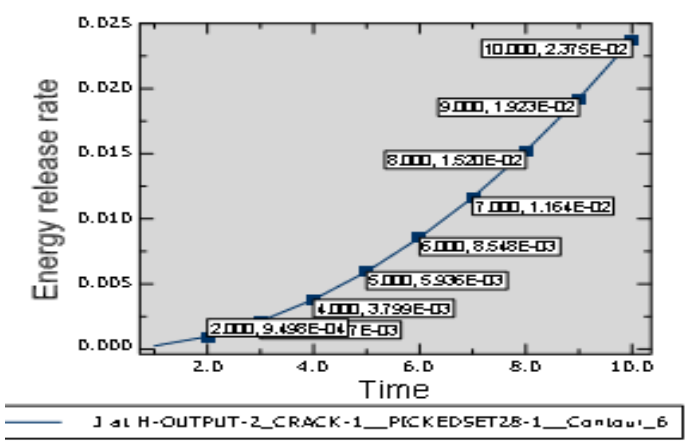

Figure.9 A curve $\mathrm{J}$ integral changes with time

By processing the $\mathbf{J}$ integral curve in Fig.9, we can get the maximum $\mathbf{J}$ integral, namely, the threshold of J integral, $J_{c}=0.02375 \mathrm{~N} / \mathrm{mm}$.

\section{Application of the XFEM to the Propellant Material in ABAQUS}

Usually using traditional ABAQUS finite element method is able to simulate the fracture behavior of propellant material. Extended finite element method is also a feature in ABAQUS6.9 version. It is needed to verified that this method weather can simulate the fracture behavior of propellant material accurately. To verify it, this paper conducted simulation aimming at viscoelastic material by XFEM.

\section{Finite Element Model and Meshing of Grids}

When the XFEM is applied to simulate the growth of crack, the crack and grids are independent. The model length is $115 \mathrm{~mm}$, and the width is $48 \mathrm{~mm}$. The crack size is $2 a=10 \mathrm{~mm}$. The loading rate is $2 \mathrm{~mm} / \mathrm{min}$. They are all same to those in Ref.[4]. The part used to simulate crack (Figure.10) and the initial setting of crack (Figure.11) are given as the figures shown. 


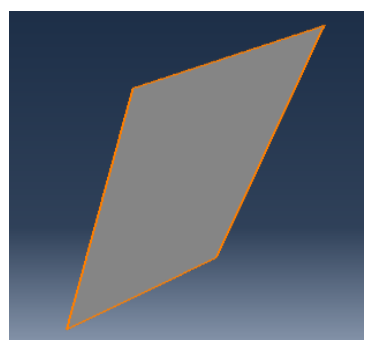

Figure.10 The part used to form crack

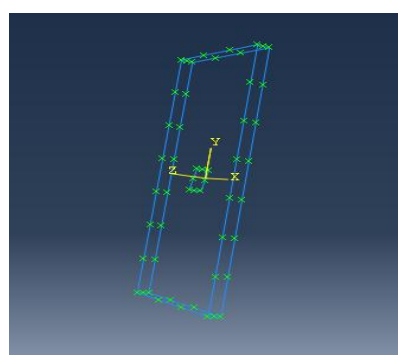

Figure.11 Distribution of the crack

This paper forms a total of 697 units using quadrilateral element and free meshing technology. From Figures.12 and 13, we can see that crack and grids are independent.

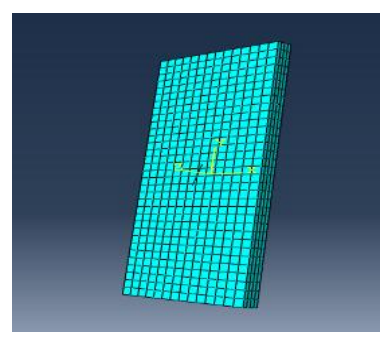

Figure.12 The whole mesh

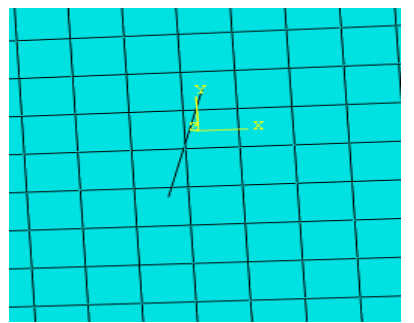

Figure.13 The grids near crack

\section{Calculation Results}

1) Crack propagation process

To display the grids failure mode in XFEM, the whole process of crack propagation results are given with grids. Before the crack propagation, mode I-II cracking happens. But with the increase of the load, it gradually transformes to mode-I cracking. In the initial stages of loading, stress concentration emerges clearly in the vicinity of the crack tip. And the crack tip begins to damage as the Figure.14 (1) shows. Then the stress of crack tip increases gradually. When the damage reaches a certain level, the initial crack start to propagate. The load near the crack tip increased gradually and crack propagates stablely. It lasts for some time as the Figure. 14 (2) shows. When the load rises to a certain extent, the cracks begin to enter the unstable state. The accelerated propagation is shown in Figure.14 (3) to Figure.14(6). This period is very short.

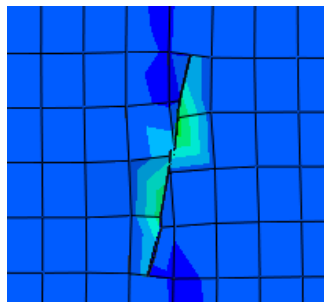

(1)

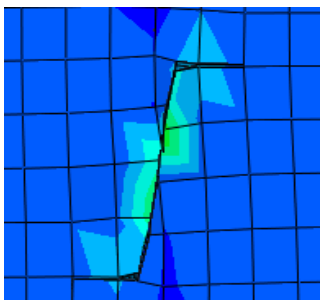

(2)

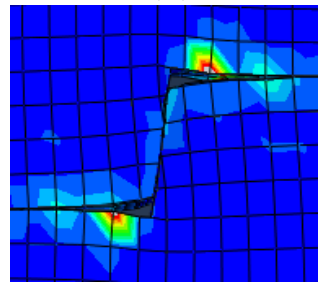

(5)

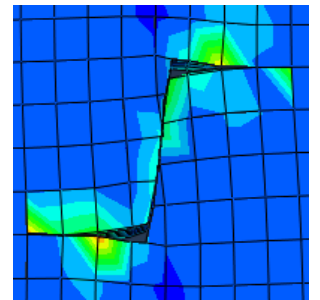

(3)

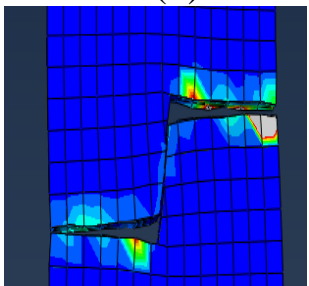

(6)

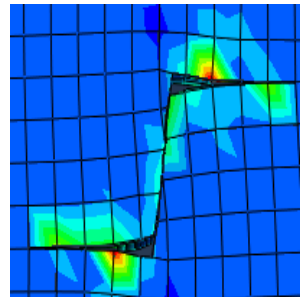

(4)

Figure.14 Crack propagation process under tensile loading

2) Comparison of the calculated results with the experimental results 
Figure.15 (2) is the state after failure and stress relaxation. Figure.15 (1) is given in Ref.[4]. From the two figures we can see that the shape of crack port and situation of crack propagation are consistent basically. It is proved that the extended finite element method is feasible for the analysis of the crack of HTPB composite propellant material.

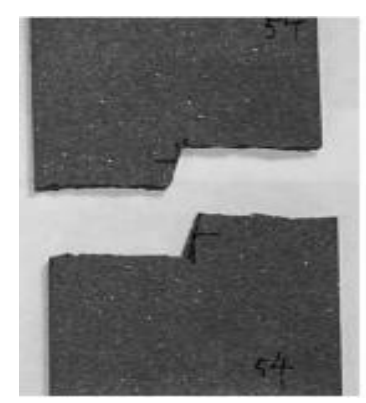

(1)[4]

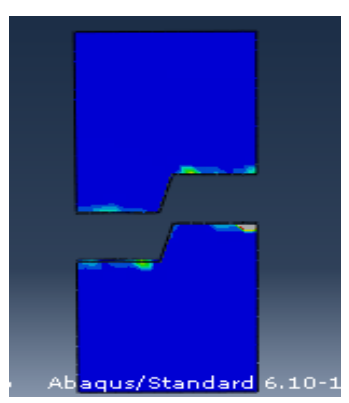

(2)

Figure.15 Comparison of the calculated results with the experimental results

In order to further prove that the extended finite element method can simulate the crack propagation of the propellant material, this paper also simulate the situation of $\beta=90^{\circ}, \beta=75^{\circ}$, $\beta=45^{\circ}$. The calculated results and the experimental results are shown as follows:

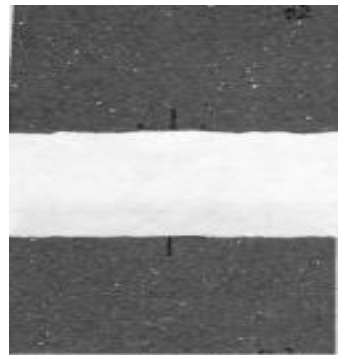

(1)[4]

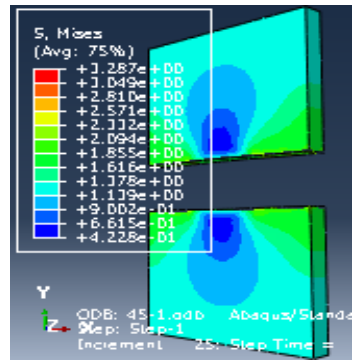

(2)

Figure.16 Crack propagation direction contrast of $\beta=90^{\circ}$

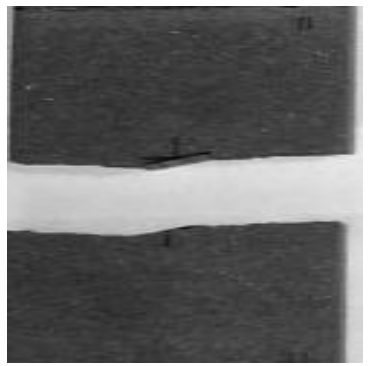

(1)[4]

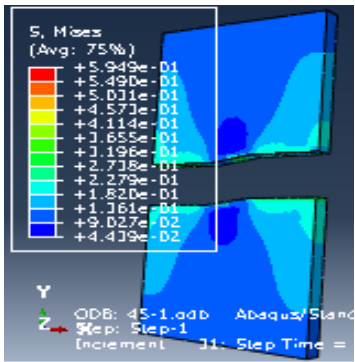

(2)

Figure.17 Crack propagation direction contrast of $\beta=75^{\circ}$

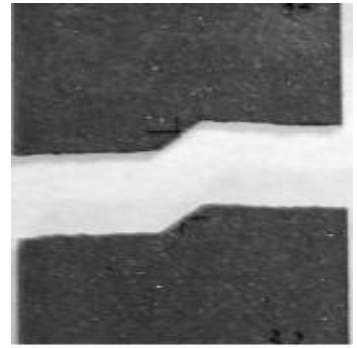

(1)[4]

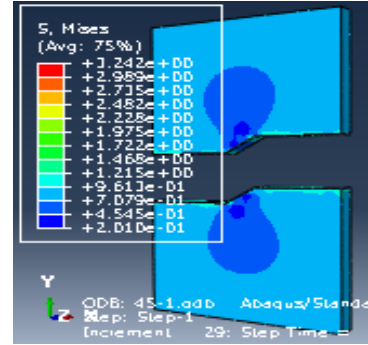

(2)

Figure.18 Crack propagation direction contrast of $\beta=45^{\circ}$ 
Under the same loading condition, the simulation results in the port of crack and the crack propagation direction are basically same with the experimental results in Ref. [4]. It is also proved that XFEM is feasible for the analysis of the crack of HTPB composite propellant material.

\section{Conclusion}

1) Using the relationship between tensile and tensile distance of Ref. [4] the paper figures out the fracture criterion of the propellant material by traditional finite element method, $J_{c}=0.02375 \mathrm{~N} / \mathrm{mm}$

2) This paper built viscoelastic model of the propellant material using XFEM. And through the comparison with the experimental results, we can see that the crack ports and crack propagation direction agree with the experimental results. It is proved that XFEM is feasible to simulate crack propagation of the propellant material.

\section{References}

[1]. Belytschko T,Black T. Elastic crack growth in finite element with minimal remeshing, Int $\mathbf{J}$ Numer Meth Eng ,1999,45:601-620.

[2].Moes N,Dolbow J,Belytschko T.A finite element method for crack growth withoutremeshing.Int J Numer Meth Eng,1999,46:131-150.

[3].Rice JR. Elastic fracture mechanics concepts for interfacial cracks. Journal of applied mechanics, 1988, 55, 98-103.

[4].ZHANG Ya,QIANG Hong-fu,YANG Yue-cheng. Fracture Behavior of HTPB Composite Propellant in 1-2 Mixed Mode Crack [J].Chinese Journal of Energetic Materials, 2007, 04: 359-362. 